\section{SOI: $1.1 /$ TAS DOI: $10.15863 / \mathrm{TAS}$ International Scientific Journal Theoretical \& Applied Science}

\author{
p-ISSN: 2308-4944 (print) e-ISSN: 2409-0085 (online) \\ Year: 2015 Issue: 04 Volume: 24
}

Published: $30.04 .2015 \quad$ http://T-Science.org

SECTION 26. Radio-technique. Electronics. Telecommunications.
Marina Yur'evna Zvezdina

Doctor of Physical and Mathematical Sciences, associate professor, head of the department, Don State Technical University, Russia, Rostov-on-Don zvezdina_m@mail.ru

Yulia Aleksandrovna Shokova Candidate of Physical and Mathematical Sciences, associate professor, Don State Technical University, Russia

Andrey Viktorovich Shokov postgraduate student, Don State Technical University, Russia

Nikolay Nikolaevich Efimenko student,

Don State Technical University, Russia

\title{
PROBABILITY PLOTS APPLICATION FOR SKILLS TRANSFER IN TESTING EXPERIMENTAL DATA FOR NORMALITY
}

\author{
Abstract: The examples of probability plots application in teaching process for students majoring in radio \\ engineering for experimental data processing, including testing for normality, are given. Probability-probability \\ and quantile-quantile plots applications are considered. Plotting algorithm and interpretation of results are given. \\ Key words: competency-based approach, experimental data processing, testing for normality, probability \\ plots, result interpretation \\ Language: Russian \\ Citation: Zvezdina MY, Shokova YA, Shokov AV, Efimenko NN (2015) PROBABILITY PLOTS \\ APPLICATION FOR SKILLS TRANSFER IN TESTING EXPERIMENTAL DATA FOR NORMALITY. ISJ \\ Theoretical \& Applied Science 04 (24): 155-161. \\ Soi: http://s-o-i.org/1.1/TAS*04(24)27 Doi: crossef http://dx.doi.org/10.15863/TAS.2015.04.24.27

\section{ПРИМЕНЕНИЕ ВЕРОЯТНОСТНЫХ ГРАФИКОВ ДЛЯ ПРИВИТИЯ НАВЫКОВ ПРОВЕРКИ НОРМАЛЬНОСТИ ЭКСПЕРИМЕНТАЛЬНЫХ ДАННЫХ}

Аннотация: Приводятся примеры использования вероятностных графиков в процессе обучения студентов радиотехнических специальностей для обработки экспериментальных данных, в частности, проверки на нормальность их распределения. Рассмотрены варианты использования вероятностновероятностных и квантиль-квантильных графиков. Приводится алгоритм построения графиков, а также дается трактовка получаемых результатов.

Ключевые слова: компетентностный подход, обработка данных эксперимента, проверка нормальности, вероятностные графики, трактовка результов.

Современный этап построения процесса обучения в учреждениях высшего образования характеризуется переходом на новые федеральные государственные стандарты высшего образования (ФГОС ВО) [1, 2]. Отличительной особенностью данных стандартов является использование компетентностного подхода, предусматривающего формирование поведенческой модели. Особенностью данной модели является способность у обучаемого анализировать возникающую задачу, выбирать возможный вариант еe разрешения, а также требуемые для этого технические средства. Подтверждением сказанного является включение в ФГОС ВО профессиональных компетенций, ориентированных на работу с информацией и моделированием процессов $[1,2]$. Так, в ФГОС ВО по направлениям подготовки 11.03.01 «Радиотехника» и 11.03 .02 «Инфокоммуникационные технологии и системы связи» данные компетенции сформулированы следующим образом: «способность реализовывать программы экспериментальных исследований, включая выбор технических средств и обработку результатов (ПК-2)» для первого направления и «способность применять современные теоретические экспериментальные методы исследования с 
целью создания новых перспективных средств электросвязи и информатики (ПК-17)» для второго направления. Анализ перечисленных выше компетенций делает особо актуальным навыки обработки данных экспериментальных исследований, включая и определение закона распределения, которому подчиняются, в частности, погрешности вычислений. Для реализации компетенций в учебном процессе целесообразно разработку модулей, посвященных статистической обработке экспериментальных данных, осуществлять с привлечением информационных технологий, позволяющих осуществить дифференциацию и индивидуализацию обучения, а также развивать творческую активность у студентов [3-6].

В качестве объекта исследований таких модулей необходимо рассмотреть определение закона распределения эмпирических данных, в частности, подтверждение гипотезы о нормальном законе распределения данных. В стандарте [7] показано, что в случае отсутствия дополнительной информации о виде эмпирического распределения, первоначально экспериментальные данные проверяются на нормальность. Для этого, как следует, например, из $[7 ; 8$, с. 94; 9, с. 231-278], применяются несколько классов методов, в том числе:

- критерии согласия;

- построение вероятностных графиков.

При этом первый класс относится к строгим методам и может быть условно назван «математическим». Второй класс, «графический», относится к нестрогим методам. В обоих случаях проверка выдвинутой гипотезы о виде распределения осуществляется путем сравнения экспериментальных данных с теоретическим распределением. Различие заключается в способе проверки гипотезы. В первом случае выбирается критерий согласия, формулируется его гипотеза, рассчитывается статистика, сравнивается с критическим значением и на основе данного сравнения делается вывод о принятии или отклонении гипотезы [9, с. 231-278]. При использовании графических методов, как следует из [7; 10, c. 143], на декартову плоскость наносятся точки, характеризующие два распределения экспериментальное и ожидаемое по теоретическому закону, т.е. графически сравниваются кумулятивные функции распределения. По виду полученного графика делается вывод о совпадении или отличии законов распределения. Если график приближается к прямой, то распределение данной переменной будет нормальным. При отличии законов имеется дополнительная информация, позволяющая продолжить процесс идентификации теоретического закона распределения.

Математические и графические классы методов определения закона распределения отличаются по сложности реализации, а выбор конкретного метода зависит от цели исследований. Так, в ряде частных практических случаев, когда не требуется строгого доказательства нормальности закона распределения, а требуется лишь его подтверждение, наиболее целесообразно использовать графические методы. Кроме того, графические методы, не содержащие в чистом виде механизма статистической проверки гипотез, являются более простыми для понимания и освоения, а, следовательно, могут быть использованы как первая ступень при изучении темы определения эмпирического закона распределения.

Следует отметить, что, несмотря на простоту реализации графических методов, а также на наличие использующего их государственного стандарта [7], в российской научной школе они, как правило, не используются.

Рассмотрим более подробно использование графических методов в процессе изучения способов проверки нормальности полученных экспериментальных данных. Для этого воспользуемся материалами из [7, 10-12].

Прежде чем приступать к построению графиков необходимо определиться со следующими вопросами:

- в зависимости от размера выборки относительно чего будет строиться кумулятивная функция - относительно накопленной частости (при небольшом размере выборки) либо накопленной вероятности (при большом размере выборки);

- какой функцией будет описываться теоретическое распределение.

В стандарте [7] показано, что графические методы базируются на построении кумулятивной кривой, т.е. кривой накопленной частости, либо при большом размере выборки - накопленной вероятности. В зависимости от того, какая кумулятивная функция выбирается - прямая или обратная - графики делятся на два типа [10, c. $142-143 ; 11$, c. $21,22,24]$ :

- Р-P графики, если в качестве характеристики сравнения выступает вероятность. В данном случае проводится сравнение функций распределения теоретического и эмпирического законов для всех значений выборки;

- Q-Q графики, если в качестве характеристики выступают квантили распределений. Для эмпирического 
распределения квантилями являются сами значения выборки.

Данные графики соответствуют методам прямого и обратного преобразования.

Алгоритм построения вероятностных графиков подробно представлен, например, в [10, c. 142-145] или [11, с. 21-24]. Рассмотрим этот алгоритм на примере проверки на нормальность эмпирических данных, представленных выборкой $x_{N}$. В соответствии с данными работами первым шагом обработки данных является построение вариационного ряда $x_{1}<x_{2}<\ldots<x_{N}$. Затем рассчитываются эмпирические вероятности $x_{1}<x_{2}<\ldots<x_{N}, \quad p_{i} . \quad$ В зависимости от конкретного значения величины $a$ используются различные алгоритмы расчета эмпирических вероятностей. Ряд из них приведен в табл.1 [11, c.19-20].

\section{Таблица 1}

Соотношения для вычисления эмпирических вероятностей различными методами.

\begin{tabular}{|c|c|c|c|c|}
\hline $\begin{array}{c}\text { Метод } \\
\text { статистической } \\
\text { обработки }\end{array}$ & $\begin{array}{c}\text { Формула для расчета } \\
\text { эмпирической вероятности } \\
p_{i}\end{array}$ & $\begin{array}{c}\text { Метод } \\
\text { статистической } \\
\text { обработки }\end{array}$ & \multicolumn{2}{|c|}{$\begin{array}{c}\text { Формула для расчета } \\
\text { эмпирической вероятности } p_{i}\end{array}$} \\
\hline Общий случай & $p_{i}=\frac{i-a}{N+1-2 a}$ & \begin{tabular}{|l} 
Метод $\quad$ рангового \\
преобразования
\end{tabular} & \multicolumn{2}{|c|}{$p_{i}=\frac{i-0,5}{N}$} \\
\hline Метод Блома & $p_{i}=\frac{i-0,375}{N+0,25}$ & \multirow[t]{3}{*}{ Метод Филлибена } & \multirow{3}{*}{$p_{i}=\left\{\begin{array}{c}1-p_{N}, \\
i-0,3175 \\
N+0,365 \\
0,5^{1 / N},\end{array}\right.$} & \multirow{3}{*}{$\begin{array}{c}i=1 \\
i=2 \ldots N-1 \\
\quad i=N\end{array}$} \\
\hline Метод Тьюки & $p_{i}=\frac{i-1 / 3}{N+1 / 3}$ & & & \\
\hline 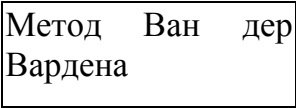 & $p_{i}=\frac{i}{N+1}$ & & & \\
\hline
\end{tabular}

Далее при построении Р-P графиков проводится расчет ожидаемых вероятностей для стандартизированного нормального распределения с использованием значений функции Лапласа $\Phi\left(z_{i}\right)$ (где $z_{i}=\frac{x_{i}-\mu}{\sigma}, \mu$ и $\sigma$ оцененные по выборке значения параметры нормального распределения). По полученным значениям на декартову плоскость наносятся точки с координатами $\left(p_{i}, \Phi\left(z_{i}\right)\right)$. Для проверки нормальности построением Q-Q графиков производится нахождение квантилей стандартизированного нормального закона распределения [10, с.21]:

$$
Q_{i}=\Phi^{-1}\left(p_{i}\right),
$$

а затем на декартову плоскость наносятся точки с координатами $\left(Q_{i}, x_{i}\right)$. При этом оценка параметров распределения не проводится, что может рассматриваться как достоинство данного типа графиков.

P-P и Q-Q графики имеют общую интерпретацию [11, с. 24]. В случае, когда набор точек на графике рассеян около прямой линии, считается, что генеральная совокупность, из которой взята выборка, подчиняется нормальному закону распределения. Если точки отклоняются от прямой систематически, то эмпирический закон распределения не является нормальным. При этом возможны следующие интерпретации данных отклонений [11, с. 21], [12]:

- отличия в асимметрии распределений;

- отличия в длине хвостов распределений, если эмпирический закон распределения симметричен;

- бимодальность.

Удлинение хвостов в графиках обусловлено расширением относительной частоты к краям, обусловленное большим рассеянием экстремальных значений по сравнению со среднеарифметическими значениями. Бимодальность графиков показывает, что выборка не является однородной и наблюдения порождены двумя или более «наложенными» распределениями. В ряде случаев бимодальность распределения означает, что выбранные инструменты не подходят для измерения.

На рис. 1-4 приведены иллюстрации возможных видов вероятностных графиков при 
отклонениях экспериментальных данных от нормального закона распределения: без отклонения (рис.1), при отклонении в асимметрии (рис.2), при отличиях в хвостах распределения (рис.3), при бимодальности распределения (рис.4). Причем на левом поле рисунков приведены квантиль-квантильные графики (Q-Q графики), а на правом поле вероятностно-вероятностные графики (Р-Pграфики). Теоретический вид графиков для данных случаев показан в правом нижнем углу поля, а вид распределения плотности вероятности - в верхнем левом углу. При этом на рис.3 точками показан нормальный закон распределения, а зеленой линией выбранный закон распределения.

Построение графиков проводилось для выборок объемом 100 значений каждая, сформированных в среде MathCad 15 с использованием датчика случайных чисел, распределенных по некоторому заданному закону. Использовались следующие законы распределения:
- нормальный закон распределения с параметрами $\mu=-5, \sigma=4$ (встроенная функция rnorm);

- экспоненциальный закон с параметром $\lambda=4$ в качестве закона с положительной асимметрией (встроенная функция rexp);

- распределение Вейбулла с параметром формы $\beta=7$ в качестве закона с отрицательной асимметрией (встроенная функция rweibull);

- равномерный закон распределения с параметрами $\mathrm{a}=-3, \mathrm{~b}=3$ в качестве закона распределения с короткими хвостами (встроенная функция runif);

- распределение Стьюдента с числом степеней свободы $\mathrm{k}=2$ в качестве закона распределения с длинными хвостами (встроенная функция rt);

- смесь двух выборок объемом 50 значений каждая, случайные величины распределены по нормальному закону распределения с параметрами $\mu 1=-1, \sigma 1=0,3$ и $\mu 2=1, \sigma 2=0,3$.

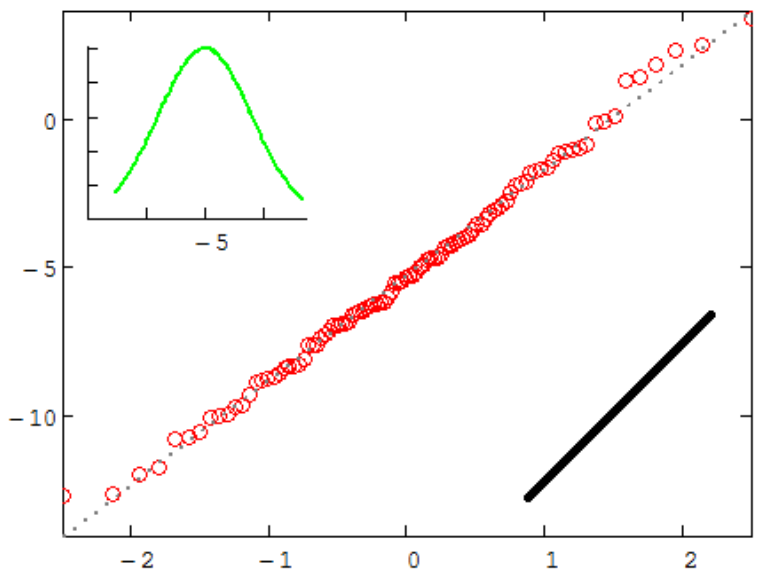

a)

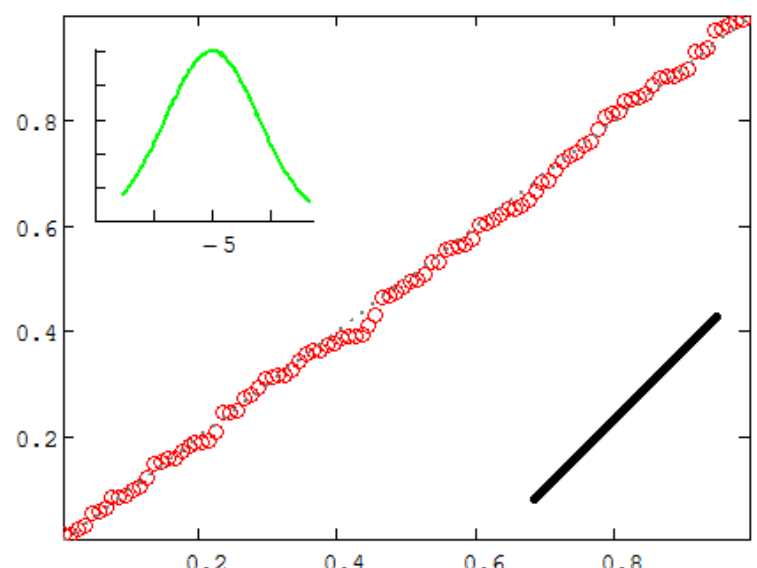

б)

Рисунок 1 - Вероятностный график при нормальном законе распределения эмпирических данных: a) для квантильного подхода; б) для вероятностного подхода.

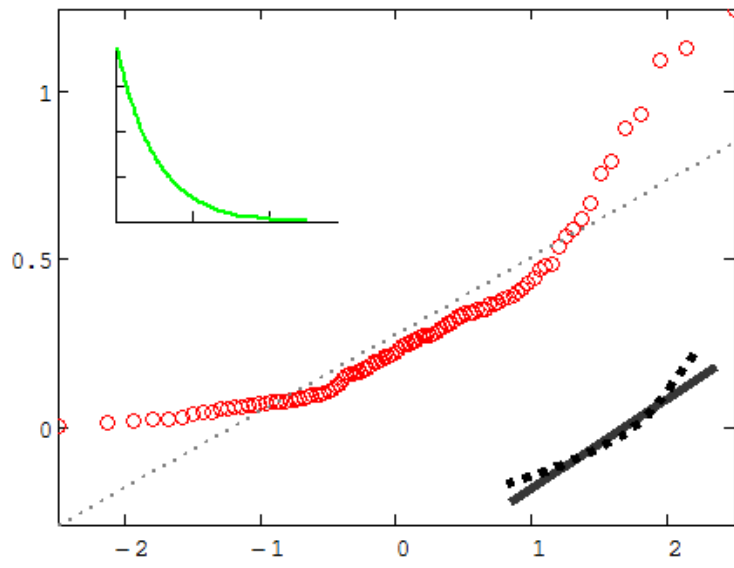

a)

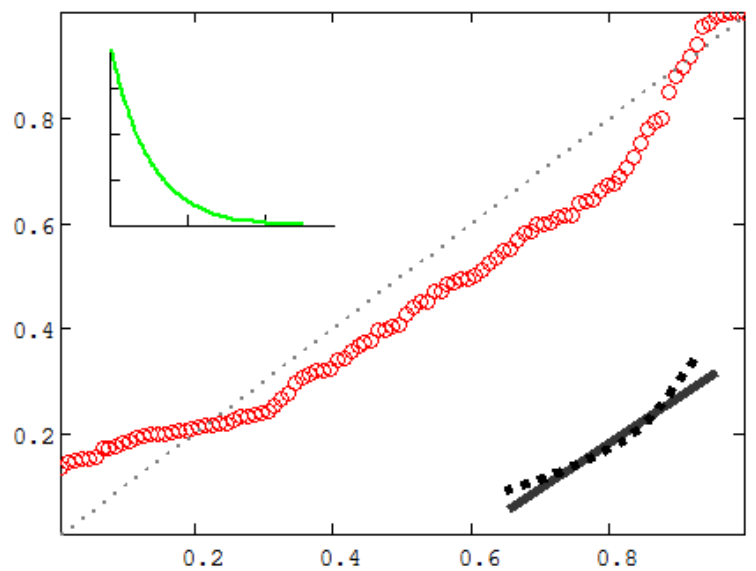

б)

ISPC The Combination of Technology \& 


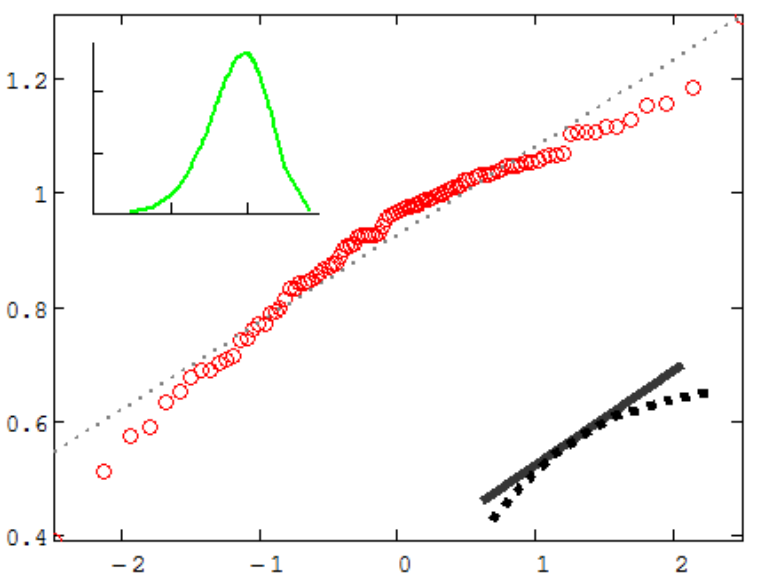

B)

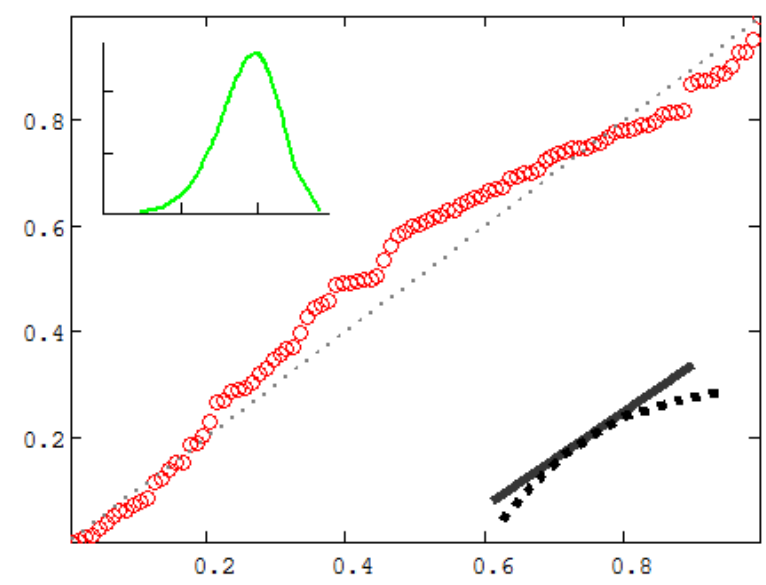

г)

Рисунок 2 - Отклонения в асимметрии на вероятностном графике: положительная - $a, \delta$, отрицательная - в,2.

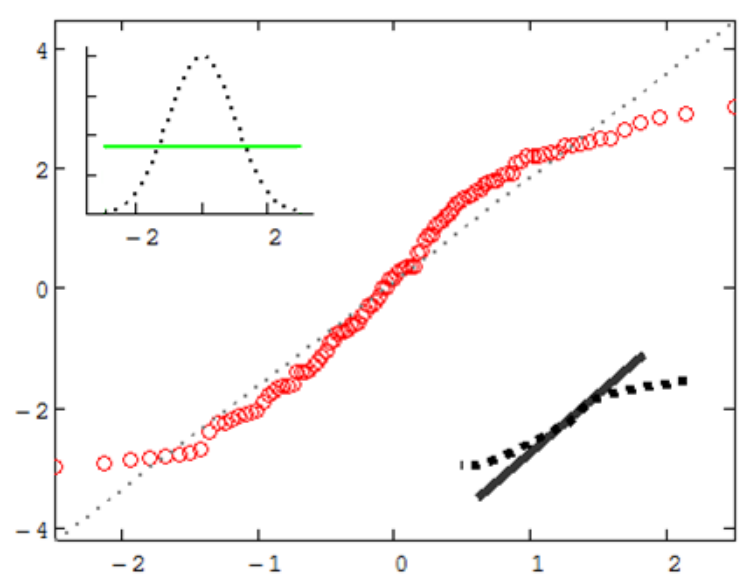

a)

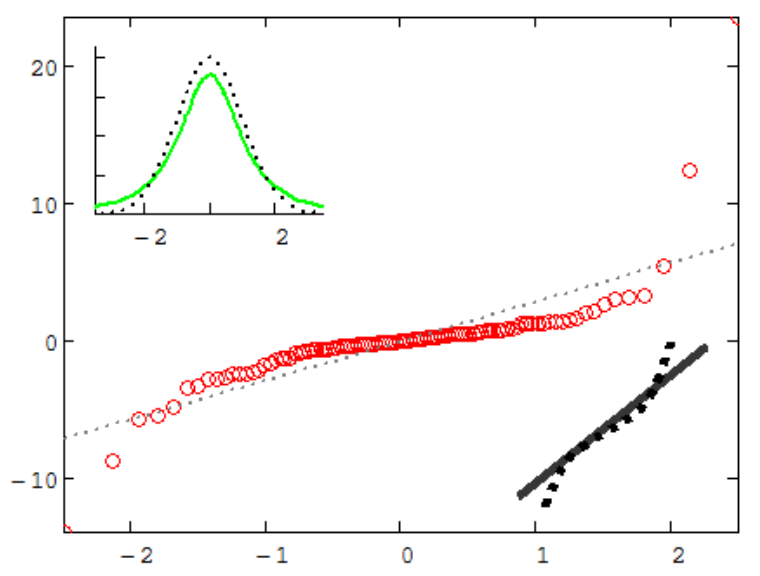

B)

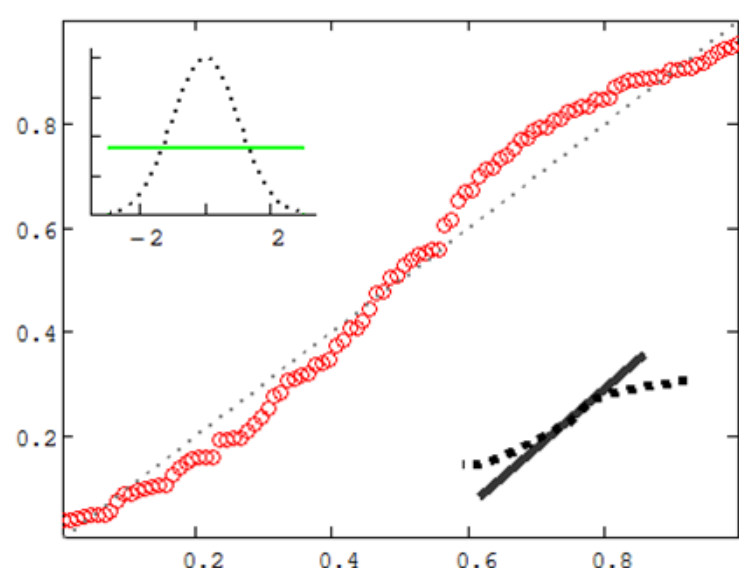

б)

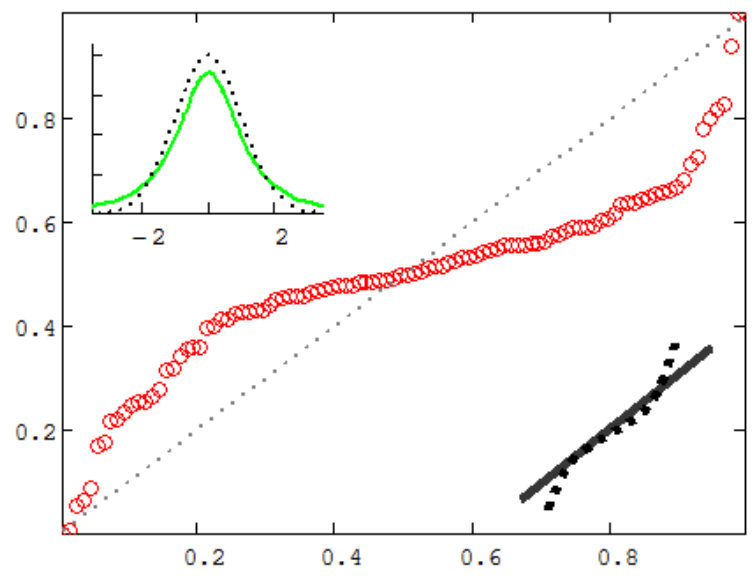

г)

Рисунок 3 - Отличия в длине хвостов на вероятностном графике: распределение с короткими хвостами - $a, \sigma$, распределение с длинными хвостами - в,2. 


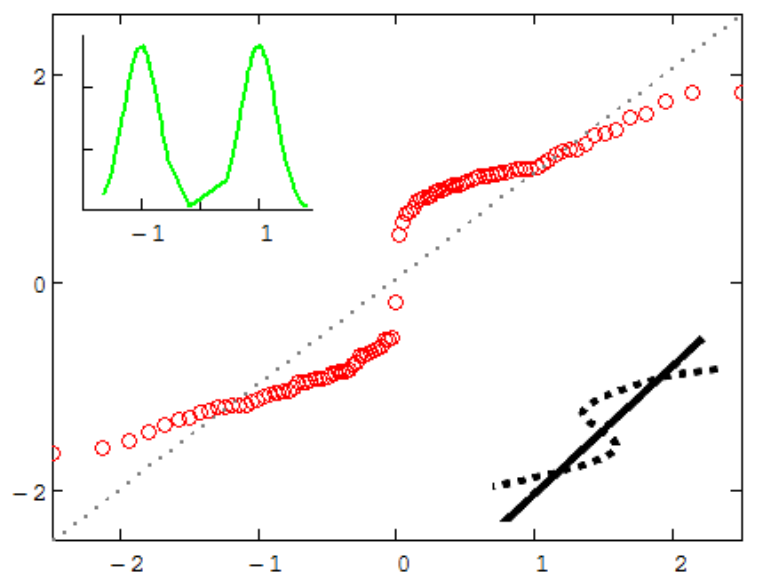

a)

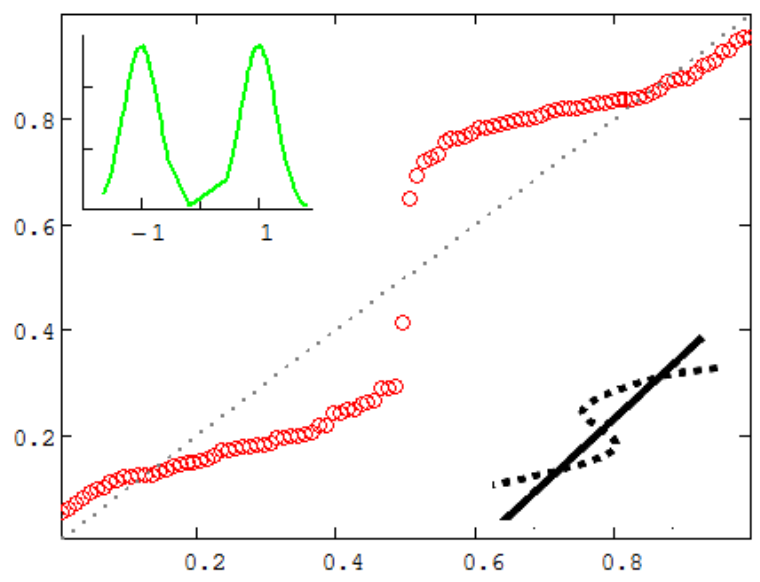

б)

Рисунок 4 - Бимодальность на вероятностном графике.

Анализ приведенных результатов показывает, что по виду графика может быть легко дана трактовка получаемых результатов (предварительное определение нормальности распределения экспериментальных данных):

- если точки выстраивают вдоль прямой линии, то экспериментальные данные распределены по нормальному закону;

- если точки выстраиваются в дугу, закон распределения данных асимметричен, при этом направление выпуклости характеризует знак асимметрии: вниз - положительная асимметрия, вверх - отрицательная;

- если изгиб кривой напоминает букву «S», то есть нижний конец графика направлен влево, верхний - вправо, и при этом в центре графика нет разрыва, то данные распределены по закону с короткими хвостами;

- если кривая представляет собой зеркальное отражение буквы «S» по вертикали, нижний конец графика направлен вниз, а верхний - вверх, то данные распределены по закону с длинными хвостами;

- если изгиб кривой напоминает букву «S» и при этом наблюдается разрыв посредине, то исходные данные бимодальны.
Данная трактовка совпадает с описаниями вероятностных графиков из [7] и Q-Q графиков из $[11$, с. 21$]$.

Сравнение вероятностно-вероятностных и кквантиль-квантильных графиков между собой показывает, что по своему виду они практически совпадают, однако наблюдаются и их отличия. Так, Q-Q графики являются более чувствительными к отличиям данных от теоретического закона распределения (обладают лучшим разрешением) на хвостах, а Р-Р графики - по центру. При этом в основном все различия сосредоточены именно на концах графиков, кроме случая бимодальности, в связи с этим для анализа графическими методами чаще всего используется именно квантиль-квантильные графики.

Таким образом, полученные результаты показывают, что графические методы могут быть использованы для привития навыков обработки экспериментальных данных при обучении по направлениям подготовки 11.03.01 Радиотехника и 11.03.02 Инфокоммуникационные технологии и системы связи.

\section{References:}

1. (2015) Federal'nyy gosudarstvennyy obrazovatel'nyy standart vysshego obrazovaniya po napravleniyu 11.03.01 Radiotekhnika (uroven' bakalavriata). Utv. Prikazom Ministerstva obrazovaniya i nauki Rossiyskoy Federatsii ot 6 march 2015. №179.

2. (2015) Federal'nyy gosudarstvennyy obrazovatel'nyy standart vysshego obrazovaniya po napravleniyu $\quad 11.03 .02$ Infokommunikatsionnye tekhnologii i sistemy svyazi (uroven' bakalavriata). Utv. Prikazom Ministerstva obrazovaniya i nauki Rossiyskoy Federatsii ot 6 march 2015. №174.

3. Ponomarev AK, Pronin AV, Starykh VA, Tikhonov AN (2012) Informatizatsiya sfery obrazovaniya i nauki Rossii: strategiya razvitiya. 
Informatizatsiya obrazovaniya i nauki, 4(16), pp. 3-26.

4. Abdugulova ZK (2014) Rol' innovatsion-nykh tekhnologiy $\mathrm{v}$ organizatsii uchebnogo protsessa $\mathrm{v}$ vuzakh. Innovatsii $\mathrm{v}$ nauke: pri-menenie $\mathrm{i}$ rezul'taty. Ezhemesyachnyy nauch-nyy zhurnal Mezhdunarodnogo nezavisimogo instituta Matematiki i Sistem, 11, pp. 4-6.

5. Uskov VL, Ivannikov AD, Uskov AV (2009) Strategiya razvitiya sistem elektron-nogo obucheniya na period do 2015 goda. Informatizatsiya obrazovaniya i nauki, 4, pp. 144158.

6. Zvezdina MY, Zaikin DS (2010) Interaktivnyy kurs obucheniya distsipline «Elektrodinamika i rasprostranenie radiovoln». V mire nauchnykh otkrytiy, 1(07), pp. 138-141.

7. (2002) GOST R ISO 5479-2002. Statisticheskie metody. Proverka otkloneniya raspredeleniya veroyatnostey ot normal'nogo raspredeleniya. Data vvedeniya 01.07.2002.

8. Lemeshko BY, Lemeshko SB, Postovalov SN, Chimitova EV (2011) Statisticheskiy analiz dannykh, modelirovanie i issledovanie veroyatnostnykh zakonomernostey. Komp'yuternyy podkhod. Novosibirsk: Izd-vo NGTU.

9. Kobzar' AI (2008) Prikladnaya matematicheskaya statistika. Moscow: Fizmatlit.

10. Gibbons JD, Chakraborti S (2014) Nonparametric Statistical Inference, Fifth Edition: Revised and Expanded. Boca Raton, Florida: CRC Press.

11. Thode HC (2002) Testing for normality, Boca Raton, Florida: CRC Press, 2002.

12. Motulsky H (2013) QQ normality plots, Available:

http://graphpad.com/faq/file/1872QQnormality plots.pdf (Accessed: 18.04.2015) 